\title{
Transmission and Reflection of Electromagnetic Waves by a Hot Plasma
}

\author{
Edward C. Taylor \\ Aerospace Corporation, Los Angeles, Calif.
}

(Received October 16, 1964; revised November 25, 1964)

\begin{abstract}
The relativistic form of the Vlasov equation is used to solve the problem of transmission and reflection of normally incident electromagnetic waves. Transmission and reflection coefficients are derived for a plasma half-space and for a plasma slab, using the assumption that electrons are specularly reflected at the boundaries. These coefficients are functions of the ratio of the electron thermal speed to the vacuum speed of light, indicating that temperature dependence in these cases is a relativistic effect. Nevertheless, it is seen that the nonrelativistic limits of these coefficients differ from those obtained by using the cold-plasma equations since the transition to the zero-temperature limit has a nonuniform character.
\end{abstract}

\section{Introduction}

Investigations of transverse electromagnetic wave propagation in isotropic plasmas are usually based on the cold-plasma equations. Since such an approach reveals that the phase velocity of the waves is larger than the vacuum speed of light $c$, and therefore much larger than physically admissible thermal velocities of the plasma particles, it is concluded a posteriori that thermal effects are negligible. The validity of the cold-plasma approximation for the case of a transverse wave propagating in an unbounded collisionless plasma has been demonstrated by Buneman, [1961a], who derived a relativistically invariant dispersion relation by solving the equations of motion and conservation of the different electron streams that are superimposed on each other in their combined fields. In contrast to the case of longitudinal waves, in which the inclusion of temperature effects is necessary to obtain a dispersion relation, Buneman's dispersion relation for transverse waves contains a thermal correction to the cold plasma result that is negligible for laboratory temperatures.

The large phase-velocity argument which motivates the use of cold-plasma formulas applies only to regions of the plasma which are far away from boundaries. In investigations of longitudinal wave problems, a kinetic approach has revealed the existence of a short range disturbance which is a superposition of modes which have phase velocities ranging from zero to the vacuum speed of light [Taylor, 1963]. It is expedient, then, to use a relativistic kinetic analysis to investigate the effect of boundaries on the transmission of transverse waves, since the slow components of the field provide a mechanism for the exchange of energy between the field and the plasma even in the absence of collisions. In this paper, the approach previously developed for longitudinal waves is extended to the transverse wave problem. We will obtain a dispersion relation for the propagating component of the field which is identical to that of Buneman, and also a tractable expression for the short range disturbance produced by the effect of the boundary on the electrons. We will then define transmission and reflection coefficients for the plasma half-space, and contrast these with the results obtained with the cold plasma approximation. Finally, we will treat the problem of electromagnetic wave propagation in a plasma slab. In both of these cases temperature dependence of the coefficients vanishes when the electron thermal velocity may be neglected when compared to the speed of light, but the limiting results differ from those obtained by using the cold-plasma equations.

It should be noted that as in most treatments of problems of this type, we will assume specular reflection of electrons at the boundary. This assumption is based on the existence of a potential sheath [Bohm and Gross, 1950].

\section{Formulation of the Problem}

We erect a rectangular coordinate system so that the plasma is contained in the half-space $z>0$. An incident transverse wave with frequency $\omega$ propagates in the vacuum in the positive $z$-direction so that its electric field is alined with the positive $x$-direction. We will determine the fields in the plasma and the amplitude of a wave reflected into the vacuum. Because we wish to solve this problem by means of a Fourier transformation, we use a mathematical artifice which was introduced by Shafranov [1958]. We consider an infinite plasma with a sheet current source at the origin $z=0$. Setting $E(z)=\hat{e}_{x} E(z) e^{-i \omega t}$, we have

$$
\frac{\partial^{2}}{\partial z^{2}} E+\frac{\omega^{2}}{c^{2}} E+i \omega \mu_{0} j=-A \delta(z)
$$

where $j$ is the plasma current and $A$ is an arbitrary constant. The solution of (1) for all $z$ is certainly a permissible solution for the half-space $z>0$ of our original problem. We need only require that at $z=0$, it connects a physically proper way to the vacuum field 
in the left half-space, which means that the constant $A$ must be chosen so that the electric and magnetic fields are continuous across the boundary.

The plasma current is given by

$$
j=e \int d^{3} u \beta c u_{x} f
$$

where $f$ is the solution of the relativistic collisionless kinetic equation [Clemmow and Willson, 1956],

$$
-i \omega f+\beta c u_{z} \frac{\partial f}{\partial z}=-\frac{e}{\mathrm{Mc}} E \frac{\partial f_{0}}{\partial u_{x}}, \beta^{2}=1+u^{2} .
$$

The notation here is the same as that used previously in the longitudinal wave problem [Taylor, 1963]. Proceeding in a straightforward manner analogous to that of the longitudinal wave treatment, and assuming specular reflection of electrons at the boundary, we obtain

$$
\begin{aligned}
\left(\frac{\partial^{2}}{\partial z^{2}}+\frac{\omega^{2}}{c^{2}}\right) E-\int_{0}^{\infty} & K_{T}\left(\left|z-z^{\prime}\right|\right) E\left(z^{\prime}\right) d z^{\prime} \\
& +\int_{0}^{\infty} K_{T}\left(z+z^{\prime}\right) E\left(z^{\prime}\right) d z^{\prime}=-A \delta(z),
\end{aligned}
$$

where

$$
\begin{array}{r}
K_{T}(x)=\frac{i \omega \omega_{p}^{2}}{c^{2}} \int_{-\infty}^{+\infty} d u_{x} \int_{-\infty}^{+\infty} d u_{y} \int_{0}^{\infty} d u_{z} \frac{u_{x}}{u_{z}} \frac{\partial f_{0}}{\partial u_{x}} \\
\exp \left(\frac{i \omega|x|}{\beta c u_{z}}\right) .
\end{array}
$$

Now we can consistently set $E(z)=E(-z)$ to obtain

$$
\left(\frac{\partial^{2}}{\partial z^{2}}+\frac{\omega^{2}}{c^{2}}\right) E-\int_{-\infty}^{+\infty} K_{T}\left(\left|z-z^{\prime}\right|\right) E\left(z^{\prime}\right) d z^{\prime}=-A \delta(z) .
$$

A Fourier transformation of (6) yields

$$
E(z)=-\frac{A}{2 \pi} \int_{-\infty}^{+\infty} \frac{e^{i k z} d k}{\frac{\omega^{2}}{c^{2}}-k^{2}-K_{T}(k)},
$$

where

$$
K_{T}(k)=\frac{\omega_{p}^{2}}{c^{2}} \int d^{3} u \frac{\beta u_{x} \frac{\partial f_{o}}{\partial u_{x}}}{\frac{k c}{\omega} \beta u_{z}-1} .
$$

We have set $\omega=|\omega| e^{i \delta}$ so that the integrand is defined everywhere in the $k$-plane except at those points on the branch line $k=|k| e^{i \delta}$ for which $|k|>\left|\frac{\omega}{c}\right|$.

We may simplify the integral in (7) by deforming the contour of integration in the $k$-plane. The result of such a procedure is that the electric field may be written as a sum of two components $E_{1}$ and $E_{2}$, where

$$
E_{1}=-i A \sum_{j} \operatorname{Res}\left[\frac{e^{-i k z}}{(\omega / c)^{2}-k^{2}-K_{T}(k)}\right]_{k=k_{j}}
$$

and

$$
E_{2}(z)=+\frac{A}{2 \pi} \int_{C} \frac{e^{i k z} d k}{(\omega / c)^{2}-k^{2}-K_{T}(k)}
$$

Each $k_{j}$ in the summation of (9) is an isolated pole of the integrand of (7), and the path $C$ proceeds clockwise around the branch line in the upper half of the $k$-plane.

\section{Dispersion Relation}

To calculate $E_{1}$ we must find the roots of the dispersion relation

$$
\frac{\omega^{2}}{c^{2}}-k^{2}-K_{T}(k)=0
$$

We set $n=k c / \omega$ to obtain

$$
1-n^{2}-\frac{\omega_{n}^{2}}{\omega^{2}} I(n)=0,
$$

where

$$
I(n)=\int d^{3} u \frac{\beta u_{x} \partial f_{0} / \partial u_{x}}{n \beta u_{z}-1} .
$$

It is shown in the appendix that this relation may be written to first order in the ratio of electron thermal energy to electron rest energy as

$$
1-n^{2}-\frac{\omega_{p}^{2}}{\omega^{2}}\left[1+\left(n^{2}-5 / 2\right) \frac{3 K T}{M^{2}}\right]=0,
$$

which is the same as that obtained for the unbounded plasma by Buneman [196la], who used a different approach. If we give $\omega_{p}$ the same small phase angle $\delta$ that we have given $\omega$, then (13) is a linear equation in $n^{2}$ with real coefficients, and it has one real root. It can be shown that no roots have been lost in the approximation by examining the exact dispersion relation given by (12). This means that in the evaluation of $E_{1}$ we need consider only one pole in the $k$ plane, and that this pole lies on the positive real or the positive imaginary axis in the collisionless limit. A simple computation yields

$$
E_{1}(z)=\frac{i A e^{i k} p^{z}}{2 k_{p}}
$$

where

$$
k_{p}=\omega / c \sqrt{\frac{1-\left(\omega_{p}^{2} / \omega^{2}\right)\left(1-\frac{5}{2} K T / \mathrm{mc}^{2}\right)}{1+\left(\omega_{p}^{2} / \omega^{2}\right)\left(K T / \mathrm{mc}^{2}\right)}} .
$$


It follows from (15) that whenever $\omega^{2}<\omega_{p}^{2} \quad\left(1-\frac{5}{2}\right.$ $K T / \mathrm{mc}^{2}$ ) we obtain a nonpropagating exponentially damped disturbance for $E_{1}$, while for $\omega>\omega_{p}^{2}\left(1-\frac{5}{2}\right.$ $K T / \mathrm{mc}^{2}$ ) we obtain a propagating unattenuated wave. This conclusion represents a relativistic correction to the well-known cold plasma result. Maxwell's equations lead to a magnetic field $B_{1}$ associated with the electric field that we have computed, and it may be written

$$
\overrightarrow{B_{1}}=\hat{\mathrm{e}}_{y} B_{1}=\hat{\mathrm{e}}_{y} \frac{i A}{2 \omega} e^{i k p^{z}}
$$

\section{Short Range Component of the Plasma Field}

We may evaluate the field $E_{2}$ by the extension of Landau's method [Landau, 1946] which was previously developed for the relativistic treatment of longitudinal waves [Taylor, 1963]. We allow $\delta$ to go to zero so that the branch cut swings onto the real axis. The integral around the cut on the real axis may be reduced to

$$
\begin{aligned}
& E_{2}(z)=-\frac{A}{2 \pi} \\
& \int_{\omega / c}^{\infty} \frac{\left[K_{-}(k)-K_{+}(k)\right] e^{i k z} d k}{\left[(\omega / c)^{2}-k^{2}-K_{-}(k)\right]\left[(\omega / c)^{2}-k^{2}-K_{+}(k)\right]},
\end{aligned}
$$

where

$$
\begin{array}{r}
K_{ \pm}(k)=\frac{\omega_{p}^{2} \omega}{c^{2}} \int_{-\infty}^{+\infty} d u_{x} \int_{-\infty}^{+\infty} d u_{y} \int_{C \pm} d_{z} \\
\frac{\beta u_{x}\left(\partial f_{0} / \partial u_{x}\right)}{k c \beta u_{z}-\omega}
\end{array}
$$

The path $C_{+}$is a semicircle in the upper half of the $u_{z}$-plane, while the path $C_{\text {- }}$ is a semicircle in the lower half of the $u_{z}$-plane. It may be shown, using the method of steepest descents that $E_{2}(z)$ and $B_{2}(z)$ have negligible amplitudes several Debye lengths away from the boundary, which means that the range of these components is proportional to the square root of the temperature of the plasma. The amplitudes of these fields at the interface may be computed by setting $z=0$ in the integrands of their representations and expanding in powers of $\left(K T / \mathrm{mc}^{2}\right)$. We obtain

$$
E_{2}(0)=0\left[\left(K T / \mathrm{mc}^{2}\right)^{1 / 2}\right]
$$

and

$$
B_{2}(0)=\frac{i A \omega_{p}^{2}}{2 \sqrt{2} \omega^{3}}+0\left[\left(K T / \mathrm{mc}^{2}\right)\right] .
$$

These results, combined with (14), (15), and (16) indicate that there is a nonuniform transition to the zero-temperature case, so that the transmission and reflection coefficients of the plasma interface differ from those obtained by the cold plasma approximation even for very small temperatures. This is due to the behavior of the short-range component of the electric field -its amplitude and its range are both proportional to $\left(K T / \mathrm{mc}^{2}\right)^{1 / 2}$, so that the magnetic field which is induced by it has a finite limit as $T \rightarrow 0$.

\section{Transmission and Reflection Coefficients for a Half-Space}

Combining the results of sections 3 and 4, we find that to zero order in $v_{\text {th }} / c$ the fields at the wall are given by

$$
E(0)=\frac{i A}{2 k_{p}}, B(0)=\frac{i A}{2 \omega}(1+\alpha),
$$

where $\alpha=\frac{\omega_{p}^{2}}{\sqrt{2} \omega^{2}}$. The parameter $\alpha$ represents the correction to the results obtained by using the coldplasma equations - the latter results are given by (2l) when $\alpha$ is set equal to zero. If we define the transmission and reflection coefficients $T$ and $R$ by the equations

$$
\begin{aligned}
& \vec{E}_{T}(z)=T E_{I} e^{i k_{p} z} \hat{e}_{z}, \\
& \overrightarrow{E_{R}}(z)=R E_{I} e^{-i k_{p} z} \hat{e}_{z},
\end{aligned}
$$

where $E_{I}$ is the amplitude of the incident electric field, $E_{T}(z)$ is the electric field in the plasma far from the boundary, and $E_{R}(z)$ is the field reflected into the vacuum, we obtain

$$
\begin{aligned}
& T=\frac{2}{1+\gamma}, \\
& R=\frac{1-\gamma}{1+\gamma},
\end{aligned}
$$

where

$$
\gamma=\sqrt{1-\left(\omega_{p} / \omega\right)^{2}}(1+\alpha) .
$$

If we define $P_{I}, P_{T}$, and $P_{R}$ as the incident, transmitted and reflected power in the electromagnetic field, we obtain

$$
\frac{P_{T}}{P_{I}}=\left\{\begin{array}{ll}
\frac{4 \gamma}{(1+\gamma)^{2}} \frac{1}{1+\alpha}, & \omega>\omega_{p} \\
0 & \omega<\omega_{p}
\end{array}\right\}
$$

and

$$
\frac{P_{R}}{P_{I}}=\left\{\begin{array}{ll}
1 & , \omega<\omega_{p} \\
\left(\frac{1-\gamma}{1+\gamma}\right)^{2} & , \omega>\omega_{p}
\end{array}\right\} .
$$


The cold plasma results are obtained by setting $\gamma=\sqrt{1-\left(\frac{\omega_{p}}{\omega}\right)^{2}}$ in (22), (23), and (24). It is interesting to note that the sum of the transmitted power and the reflected power is not equal to the incident power when $\omega>\omega_{p}$, indicating that some energy is delivered to the electrons in the plasma. The fraction of incident power delivered to the plasma may easily be shown to be $(4 \gamma \alpha)(1+\gamma)^{-2}(1+\alpha)^{-1}$. (The concept of kinetic power has been discussed by Buneman [1961b] and by authors listed therein.)

\section{Transmission and Reflection Coefficients for a Plasma Slab}

In sections 3 and 4 we found a solution to the oneboundary problem in which there is no wave incident on the boundary from within the plasma. We can generalize this solution by adding the solution $\widetilde{E}$ of the equations

$$
\begin{gathered}
-i \omega \tilde{f}+\beta c u_{z} \frac{\partial \tilde{f}}{\partial z}=-\frac{e}{\mathrm{mc}} \tilde{E} \frac{\partial f_{0}}{\partial u_{x}}, \\
\tilde{J}=e \int d^{3} u \beta c u_{z} \tilde{f}
\end{gathered}
$$

and

$$
\frac{\partial^{2}}{\partial z^{2}} \tilde{E}+\omega^{2} / c^{2} \tilde{E}+i \omega \mu_{0} \tilde{j}=0
$$

which is consistent with the requirement of specular reflection. It is easily shown that such a solution is given by an electric field which is proportional to $\cos k_{p} z$, where $k_{p}$ is defined by (15). Several Debye lengths from the wall, where the short range component of the fields can be neglected, the general fields are given by

$$
\begin{gathered}
E(z)=(A+B / 2) e^{i k_{p} z}+B / 2 e^{-i k} p^{z} \\
B(z)=k_{p} / \omega\left[(A+B / 2) e^{i k} p^{z}-B / 2 e^{-i k} p^{z}\right]
\end{gathered}
$$

where $A$ and $B$ are arbitrary constants. At the boundary, where the short range component must be included, the fields are given by

$$
\begin{aligned}
& E(0)=A+B \\
& B(0)=k_{p} / \omega A(1+\alpha) .
\end{aligned}
$$

If we write the vacuum field as

$$
E_{I} e^{i k_{0} z}+E_{R} e^{-i k_{0} z}
$$

and the field inside the plasma as

$$
E(z)=E_{P I} e^{-i k} p^{z}+E_{P R} e^{+i k} p^{z},
$$

the boundary conditions become

$$
\begin{aligned}
& E_{P R}=\frac{\gamma-1}{1+\gamma} E_{P I}+\frac{2}{1+\gamma} E_{I}, \\
& E_{R}=\frac{2 \gamma}{1+\gamma} E_{P I}+\frac{1-\gamma}{1+\gamma} E_{I} .
\end{aligned}
$$

If we use these boundary conditions to solve the problem of transmission by a plasma bounded by two specularly reflecting walls of separation $d$, and then define $E(z)=E_{I} e^{i k_{0} z}+R E_{I} e^{-i k_{0} z}, \quad z<0$ and $E(z)$ $=T E_{I} e^{i k_{0} z}, z>d$, we find

$$
\begin{array}{r}
T=\frac{4 \gamma}{(1+\gamma)^{2} e^{-i k} p^{d}-(1-\gamma)^{2} e^{i k} p^{d}} \\
R=\frac{1-\gamma}{1+\gamma}\left(1-T e^{i k p^{d}}\right) .
\end{array}
$$

The ordinary cold-plasma results are again obtained by setting $\alpha=0$, which leads to $\gamma=\sqrt{1-\omega_{p}^{2} / \omega^{2}}$. It can easily be verified that $|T|^{2}+|R|^{2}=1$, indicating that there is no net exchange of energy between the field and the electrons in the zero temperature limit, but that the zero-temperature limit of transmission and reflection coefficients for the slab obtained from kinetic theory differ from those obtained from the cold plasma formalism due to the presence of $\alpha$ in the definition of $\gamma$.

\section{Discussion}

We have explored the consequences of the relativistic form of the Vlasov equation when specular reflection of electrons at boundaries is assumed. This problem has been formulated for a half-space by Felderhof [1963], who used a nonrelativistic normal mode analysis of the type introduced by Van Kampen for the study of longitudinal waves. Although Felderhof did not obtain results in closed form, he was able to conclude that the electromagnetic field can exchange energy with the plasma electrons, and that there is an unattenuated propagating wave in the plasma at sufficiently high frequencies, as we have seen in section 5. When the problem is solved for the plasma slab, however, we have seen that the net energy exchange between the field and the electrons is zero as long as the plasma is rigorously collisionless.

It is interesting to note that the actual temperature dependence of our coefficients is of order $\left(K T / \mathrm{mc}^{2}\right)^{1 / 2}$, so that temperature effects are usually negligible, but that the transition to the zero temperature limit is nonuniform.

It can easily be shown that the coefficients of transmission and reflection for a plasma in a uniform magnetic field are the same as those we have derived if the electric field vector of the incident wave has the same orientation as the fixed magnetic field. 
Part of this work was performed while the author was a graduate student at the University of California, Los Angeles. It is a pleasure to acknowledge stimulating discussions with Professor Burton D. Fried.

\section{Appendix}

To obtain the approximate eq (13) from the exact eq (12), we must evaluate the expression $I(n)$. The technique given here is a generalization of the work of Clemmow and Willson [1956] for the case of longitudinal waves. Transforming to spherical coordinates, we have

$I=\int_{0}^{\infty} d u \int_{0}^{\pi} d \theta \int_{0}^{2 \pi} d \phi \frac{u^{3} \sin ^{3} \theta \cos ^{2} \phi}{n \beta u \cos \theta-1} \beta \frac{d f_{0}}{d u}$.

Now we make the transformation $\xi=\cos \theta$ to obtain

$$
I=\pi \int_{0}^{\infty} d u u^{2} \frac{d f_{0}}{d u} \int_{-1}^{1} d \xi \frac{1-\xi^{2}}{n \xi-1 / \beta u} .
$$

We define the functions

$$
\begin{gathered}
2 h(u)=\int_{-1}^{1} \frac{d \xi}{n \xi-1 / \beta u} \\
2 g(u)=\beta^{2} u^{2} \int_{-1}^{1} d \xi \frac{\xi^{2}}{n \xi-1 / \beta u}
\end{gathered}
$$

and obtain

$$
\begin{aligned}
\frac{d}{d u} h(u) & =\frac{-\beta}{1+u^{2}\left(1-n^{2}\right)} \\
\frac{d}{d u} g(u) & =\frac{\beta^{3} u^{2}}{1+u^{2}\left(1-n^{2}\right)} .
\end{aligned}
$$

Now we define

$$
\begin{aligned}
& \frac{d}{d u} H(u)=u^{2} \frac{d f_{0}(u)}{d u} \\
& \frac{d}{d u} G(u)=\left(1+u^{2}\right) \frac{d f_{0}(u)}{d u} .
\end{aligned}
$$

so that

$$
I=2 \pi \int_{0}^{\infty} d u\left[\left(\frac{d}{d u} H(u)\right) h(u)+\left(\frac{d}{d u} G(u)\right) g(u)\right] .
$$

Integration by parts leads to

$$
I=2 \pi \int_{0}^{\infty} d u \frac{\left(1+u^{2}\right) H(u)-u^{2} G(u)}{\left(1+u^{2}\right)^{3 / 2}\left[1+\left(1-n^{2}\right) u^{2}\right]} .
$$

Now we take the electron distribution function to be

$$
f_{0}=\left(\frac{\lambda}{2 \pi}\right)^{3 / 2} \exp \left(-\frac{\lambda u^{2}}{2}\right), \lambda=\frac{\mathrm{mc}^{2}}{K T} .
$$

Expanding the denominator of the integrand and retaining the first two terms we have

$$
\left.\begin{array}{rl}
I=2 \pi \int_{0}^{\infty} d u[H(u) & {\left[1+\left(n^{2}-3 / 2\right) u^{2}\right]} \\
& -u^{2} G(u)\left[1+\left(n^{2}-5 / 2\right) u^{2}\right]
\end{array}\right] .
$$

Partial integration and use of (A5) lead to

$$
I=4 \pi \int_{0}^{\infty} d u f_{0}(u)\left[u^{2}+\left(\frac{n^{2}}{3}-5 / 6\right) u^{4}\right] \cdot
$$

Evaluating the integrals we have

$$
I=1+\left(n^{2}-5 / 2\right) \frac{3 K T}{\mathrm{mc}^{2}} .
$$

and insertion of this expression into (12) yields eq (13).

\section{References}

Bohm, D., and E. P. Gross (1950), Effects of plasma boundaries on plasma oscillations, Phys. Rev. 79, 992-1001.

Buneman, O. (1961a), Transverse waves and plasma vortices in plasma physics, ed. J. Drummond, pp. 143-166 (McGraw-Hill Book Co., Inc., New York, N.Y.).

Buneman, O. (1961b), Electron stream model of a plasma, and the problem of energy conversation in landau damping, in Radiation and Waves in Plasmas, ed. M. Mitchner, pp. 54-65 (Stanford University Press, Stanford, Calif.).

Clemmow, P. C., and A. J. Willson (1956), The dispersion equation in plasma oscillations, Proc. Roy. Soc. (London), A237, 117-131.

Felderhof, B. U. (1963), Theory of transverse waves in Vlasovplasmas, Physica 29,662-674.

Landau, L. (1946), On the vibrations of the electronic plasma, J. Physics, USSR X, 25-34.

Shafranov, V. D. (1958), Propagation of an electromagnetic field in a medium with spatial dispersion, JETP (USSR) 6, 1019-1031.

Taylor, E. C. (1963), Excitation of longitudinal waves in a bounded collisionless plasma, Phys. Fluids 6, 1305-1312. 